\title{
Impact of Penetration of Non-Synchronous Generators on Power System Dynamics
}

\section{Document Version}

Accepted author manuscript

Link to publication record in Manchester Research Explorer

\section{Citation for published version (APA):}

Papadopoulos, P. N., \& Milanovic, J. (2015). Impact of Penetration of Non-Synchronous Generators on Power System Dynamics. In host publication

\section{Published in:}

host publication

\section{Citing this paper}

Please note that where the full-text provided on Manchester Research Explorer is the Author Accepted Manuscript or Proof version this may differ from the final Published version. If citing, it is advised that you check and use the publisher's definitive version.

\section{General rights}

Copyright and moral rights for the publications made accessible in the Research Explorer are retained by the authors and/or other copyright owners and it is a condition of accessing publications that users recognise and abide by the legal requirements associated with these rights.

\section{Takedown policy}

If you believe that this document breaches copyright please refer to the University of Manchester's Takedown Procedures [http://man.ac.uk/04Y6Bo] or contact uml.scholarlycommunications@manchester.ac.uk providing relevant details, so we can investigate your claim.

\section{OPEN ACCESS}




\title{
Impact of Penetration of Non-Synchronous Generators on Power System Dynamics
}

\author{
Panagiotis N. Papadopoulos, Member IEEE, Jovica V. Milanović, Fellow, IEEE \\ School of Electrical and Electronic Engineering \\ The University of Manchester \\ Manchester, UK \\ panagiotis.papadopoulos@manchester.ac.uk, jovica.milanovic@manchester.ac.uk
}

\begin{abstract}
Due to the integration of Distributed Energy Resources (DERs), both non-synchronous generators and energy storage technologies, the dynamic behavior of power systems is changing. The extent of the impact is yet to be fully investigated and quantified. Proper models, suitable for large scale studies but also representing all the DER control and protection mechanisms that affect the dynamic behavior of individual components and the system as a whole are used in this study. The effect of DER location, penetration level and decommissioning of conventional generators is studied. A Monte Carlo approach is used to account for uncertainties resulting from different fault location, duration and loading of the system. The focus of the paper is to illustrate the effect of DERs on power system stability and dynamic signature.
\end{abstract}

Index Terms - distributed energy resources, Monte Carlo, power system dynamic signature, power system stability.

\section{INTRODUCTION}

The increasing penetration of Distributed Energy Resources (DERs), both non-synchronous generators and energy storage technologies, is raising a series of questions concerning the operation and security of power systems. DERs are usually based on relatively new technologies that influence the dynamics of conventional power systems in ways that have not yet been clearly defined [1], [2]. Since conventional generators tend to be displaced by DERs, power systems are driven closer to the stability margin rendering preventive and corrective control very important. Therefore, the need for fast and accurate methods for the online identification of power system dynamic signatures with increased DER penetration becomes very significant [3], [4].

In order to study the impact of DERs on the transmission network stability, proper models for DER units need to be implemented. The control and protection equipment operation that affect the dynamic behavior of DERs need also to be taken into consideration. Recently [5]-[7] have provided guidelines for large scale modelling of Wind Generator (WG) parks. The same is done in [2], [8], [9] for PhotoVoltaics (PVs). Since, the main types of DER units and the ones with the largest penetration are WGs and PVs, Type 3 and Type 4 WG models are used according to [2], [5]-[9].

In [1], [9] the impact of converter connected DERs on small signal stability of the system is studied by observing the changes in the frequency and damping of modes. In [2] and [10] transient stability issues are also addressed for converter connected units and WGs, respectively.

The main focus of this study is to investigate the influence of both types of these non-synchronous generators on the transmission system stability and identify the impact they have on the dynamic signature of power systems. A measurement based approach is followed in this paper and therefore all indices used to determine the impact of DERs is based on measured responses. The factors that influence the dynamic signatures are identified and the extent of the influence is quantified using appropriate indices. Several factors are investigated, such as the DER type and location, the penetration level, as well as the decommissioning of conventional units. A Monte Carlo approach is used in order to study the effect of these uncertain factors on system stability in a probabilistic manner for different fault location, duration and system loading as well as to identify changes in generator groupings when DERs are added.

\section{Methodology}

Initially three commonly used indices for assessment of system transient behaviour are used to quantify the impact of DERs on system dynamic performance. Following this probabilistic Monte Carlo studies are performed to generalize the results and to determine the impact of DER penetration level on coherency of generator responses after a fault. The influence of penetration level of DERs, type and location of DERs, loading of the network and decommissioning of conventional units are all considered in assessment.

\section{A. Quantification of DER impact on system stability}

In order to determine the extent of the impact of DERs on system dynamic responses three different indices are used 


\section{$>$ ACCEPTED VERSION OF THE PAPER <}

The first two indices namely, the maximum rotor angle deviation of each generator from the steady state and the Transient Stability Index (TSI) as defined in [10] are relevant for the assessment of non-oscillatory behaviour of generators. These two indices are defined by Eq. (1) and (2), respectively.

$$
\Delta \delta=\left|\delta_{g \max }-\delta_{i}\right|
$$

where $\delta_{\text {gmax }}$ is the maximum rotor angle of each generator observed during the disturbance and $\delta_{i}$ is the initial pre-fault rotor angle.

$$
T S I=100 \cdot \frac{360-\delta_{\max }}{360+\delta_{\max }}
$$

where $\delta_{\max }$ is the maximum rotor angle deviation between any two generators in the system.

The TSI is a global index for each contingency studied and it can provide a quick overview of the stability status of the system. Negative values for TSI mean that the system is unstable. The more positive the value of TSI is the more stable the system is considered to be.

Apart from these two indices the settling time of the rotor angle of each generator is considered as an indication of oscillatory stability. Each generator's rotor is considered to settle to a new steady state after the magnitude of oscillations become smaller than $5 \%$ of the initial rotor angle of each generator.

\section{B. Generalization of observed behaviour}

By observing the three indices mentioned above while varying the factors that are considered in this study, the main impact of DERs can be identified. In order to generalize the results for different fault location and duration, Monte Carlo studies are carried out to ensure the results can be extended for the whole network. A total duration of $20 \mathrm{sec}$ is maintained for all simulations. The number of unstable cases observed (negative TSI), as well as the number of cases where any generator is still oscillating after $20 \mathrm{sec}$ are used to determine the extent of the impact of DERs.

Hierarchical clustering is applied to obtained rotor angles from Monte Carlo simulations to determine the generator groupings and therefore the dynamic signature of a power system after a fault is cleared [4]. The uncertainty of the system loading is also considered for this case. The obtained groupings provide an insight in how the power system dynamic signature changes when DERs are connected to a network.

\section{TEST SYSTEM}

The test system used in this study is the 16 machine, 68 bus reduced order equivalent model of the New England Test
System and the New York Power System (NETS - NYPS) adopted from [3], [11], [12]. Models of two types of DER units are added to the network, as shown in Fig.1: Type 3 Doubly Fed Induction Generators (DFIGs) and Type 4 Full Converter Connected (FCC) units, which are used to represent any type of converter connected unit, such as PhotoVoltaic (PV) plants. The network is simulated in DIgSILENTPowerFactory [13]. Several individual DER units of each type are aggregated and two single aggregate generator models are connected at each bus where DER are assumed to be connected. One representing DFIG and one FCC DER so that the mix between the two types can be adjusted. The standard mix used in all cases is $66.67 \%$ DFIGs and $33.33 \%$ FCC units. For both, the DFIGs and FCC equivalent generators a $2 \mathrm{MW}$ unit model is used and the number of individual generators in the equivalent DER model is increased according to the operating scenario and consequently the level of DERs penetration.

\section{A. Conventional network}

The conventional part of the network consists of 16 synchronous generators (G1-G16) in five interconnected areas. NETS consists of G1 to G9, NYPS of G10 to G13 and three other areas are represented by G14, G15 and G16 respectively. Standard $6^{\text {th }}$ order dynamic models are used to represent all synchronous generators. G1-G8 as well as G10G16 are equipped with slow IEEE type DC1A dc exciters while G9 is equipped with a fast acting static exciter type IEEE ST1A as well as a Power System Stabilizer (PSS). All generators are also equipped with generic governors. More information on the system is available in [3], [11], [12].

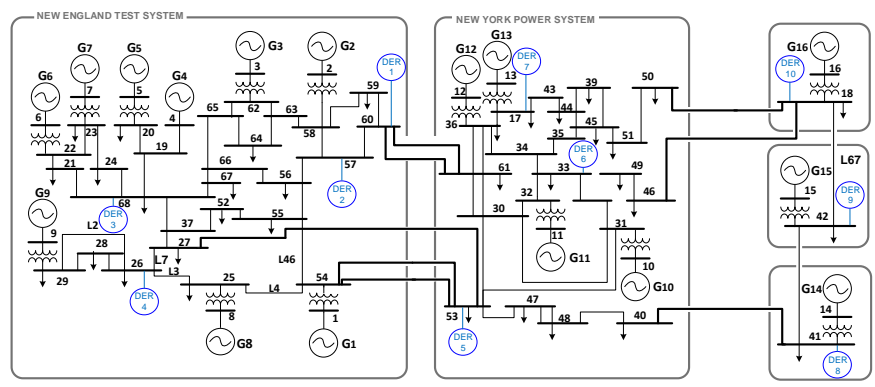

Figure 1. 68 bus test network.

\section{B. $\quad$ Type 3 DFIG modelling}

Type 3 WGs are Doubly Fed Induction Generators (DFIGs) which is the most common type especially for large WGs. The model used for DFIGs is a generic model suitable for system stability studies, which is available in DIgSILENTPowerFactory software [13] and shown in Fig. 2. The modelling approach is similar to that proposed by WECC [5] and IEC [6] working groups that developed models suitable for large scale system stability studies. The model takes into consideration the aerodynamic part and the shaft of the wind turbine/generator as well as the pitch control of the blades. The rotor side converter controller is also modeled including relevant limitations and protection mechanisms, such as the crowbar. The DFIG is represented by a typical $2^{\text {nd }}$ order model of an induction machine neglecting the stator transients and including the mechanical equation [14]. The rotor side 


\section{$>$ ACCEPTED VERSION OF THE PAPER <}

converter is controlling the voltage in the rotor [15]. Therefore, the model represents all the relevant parts that influence the dynamic behavior of DFIGs.

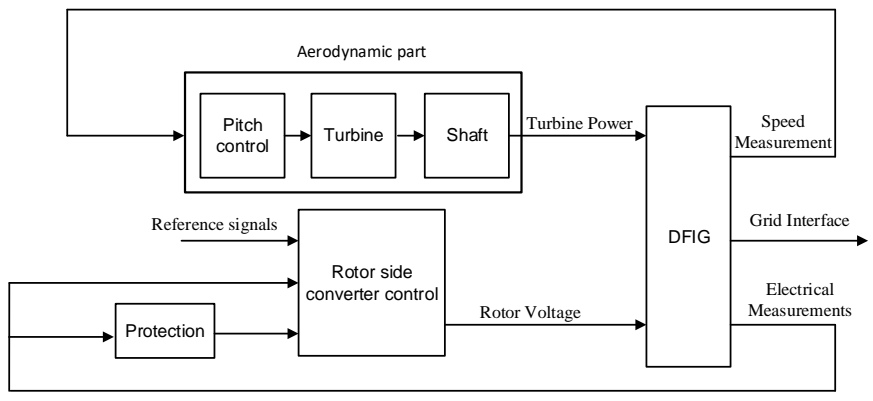

Figure 2. DFIG Type 3 model schematic.

\section{Type 4 FCC unit modelling}

Type 4 WGs are Full Converter Connected (FCC) wind generators but this model can be also used to represent any unit connected via a full converter interface, such as PVs, for system stability studies. This is possible since the converter interface is considered to decouple the dynamics of the unit behind the dc bus [1], [2], [8]. The FCC generator model, shown in Fig. 3, used in this study is available in DIgSILENT-PowerFactory software [13] and it is based on the Type 4 model presented in [6], [8]. The model is taking into consideration the converter controller with all associated limitations and also provides the ability to include frequency droop.

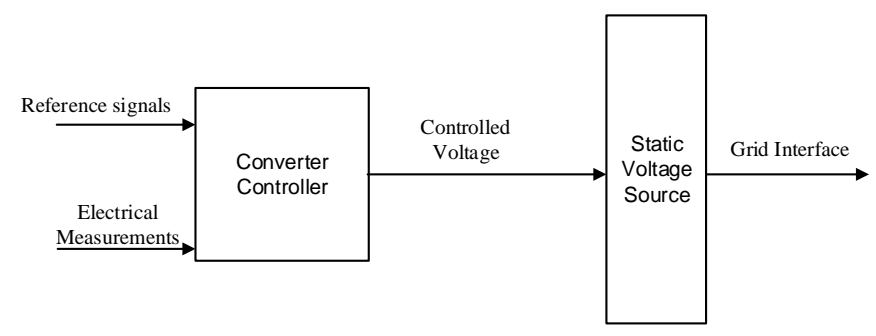

Figure 3. FCC unit model schematic.

\section{IMPACT ON SYSTEM STABILITY}

In order to define the scenarios studied, several parameters are introduced and defined in (3)-(5) for each of the five areas of the network $(i=1 \ldots 5)$.

$$
\begin{gathered}
P L_{i}=T D G_{i} / T G_{i} \\
S C_{i}=T C G_{i} / T N C G_{i} \\
T C G_{i}=\left(1-P L_{i}\right) \cdot T G_{i}
\end{gathered}
$$

Where $T G_{i}$ is the Total Generation of the entire network for the specific operating point, $T C G_{i}$ is the Total Conventional Generation for the specific operating point, $T_{N C G}$ is the Total Nominal Conventional Generation, which is the nominal capacity of all conventional generators in the area and $T D G_{i}$ is the total DER Generation for the specific operating point. Using these parameters the Penetration Level $\left(P L_{i}\right)$ of DERs and the Spare Capacity $\left(S C_{i}\right)$ of conventional generators are defined by (3) and (4), respectively and $T C G_{i}$ is defined by (5).

\section{A. Effect of penetration level and decommissioning}

The impact of the penetration of DERs on system stability is investigated using two Test Cases (TCs) which are compared between each other and with the base case. The base case is considering only conventional generation with power flow conditions as described in [11], [12] and $S C_{i}$ of $15 \%$. In all studied cases in this section, a three phase $100 \mathrm{~ms}$ self clearing short circuit is applied to the terminals of G1.

If only the $P L_{i}$ is changed in one area of the network then this means the $T C G_{i}$ will vary according to (5) and therefore the $S C_{i}$ will change as shown in (4). This is equivalent to only de-loading generators in the network areas and not decommissioning any conventional generation.

To account for decommissioning of conventional units, the TNCGi can be varied. Since the generators are equivalent generators, reducing the nominal power and retaining all the per unit values of reactances as well as the inertia constant, is equivalent to a reduction in the actual inertia of the power plant and an increase in the reactances. In this way, the disconnection of units within a power plant is considered to account for the increase of the penetration of DERs by scaling the generators nominal characteristics. In the examined cases, $S C_{i}$ is kept constant at $15 \%$ which means that for a larger amount of penetration a larger amount of conventional units are decommissioned.

Therefore, the resulting TCs are defined as follows: TC1 has $P L_{i}=20 \%$ and $T N C G_{i}$ remains constant compared to the base case, leading to increased $S C_{i}$ in all areas of the network. TC2 has $P L_{i} 20 \%$ and $T N C G_{i}$ is reduced (by approximately $20 \%$ ) to keep $S C_{i}$ constant at $15 \%$ in all areas of the network. Accordingly, TC3 has $P L_{i} 30 \%$ and TC4 $P L_{i} 30 \%$ and $S C_{i}$ $15 \%$.

In Fig. 4 and 5, the maximum rotor angle deviation and the settling time for the above cases are presented respectively. It is concluded that when generators are only de-loaded to account for DER penetration, the maximum rotor angle deviation for all generators is reduced significantly leading to an increase in system stability. However, when conventional units are decommissioned, the positive effect of DERs, while it still exists, is much lower. This means that there are two opposing effects, one positive and one negative which both determine the final effect of DERs on system stability.

Furthermore, by observing the settling time it is noticed that as the DER penetration increases, even without decommissioning, the settling time slightly increases for some of the generators (G10-G12), pointing to possible oscillatory instability issues. This effect is more evident when conventional units are also decommissioned. The threshold where oscillatory instability for the specific operating conditions was noticed is $40 \% P L_{i}$ when units where decommissioned and $60 \% P L_{i}$ without decommissioning. It 


\section{$>$ ACCEPTED VERSION OF THE PAPER <}

should also be noted that significant changes in power flows on tie lines where noticed for high penetration levels, which could lead to changes in the inter-area modes damping.

Similar cases with $30 \%$ loading of the system are studied and the results are in accordance to the ones previously described. The only exception is that for the cases when decommissioning is studied, the $S C_{i}$ cannot be held fixed to $15 \%$ because that requires a significant reduction, in the order of $70 \%$, in the system inertia which leads to non-oscillatory instability. It was observed that inertia reduction around 50\%, even for the case of low loading, could lead to instability.

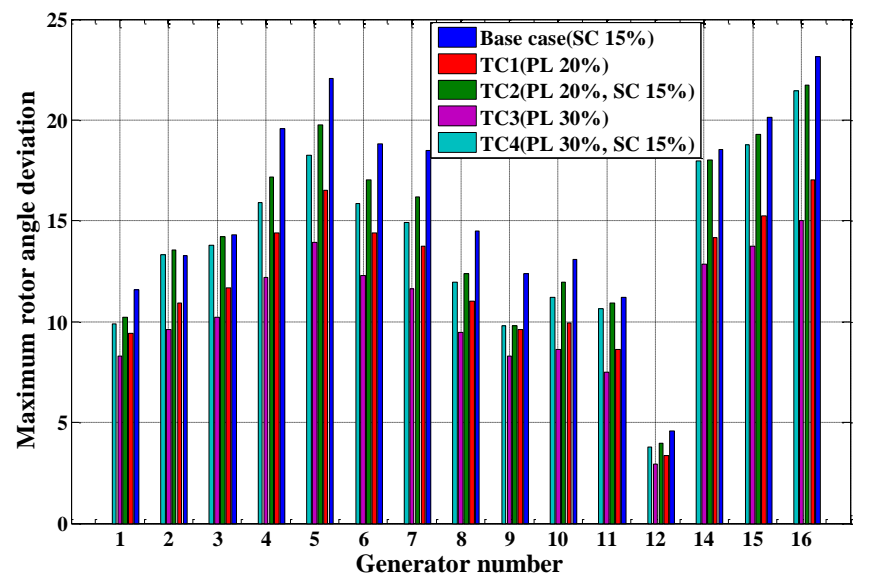

Figure 4. Maximum rotor angle deviation considering different DER penetration and decommissioning (TC1-TC4).

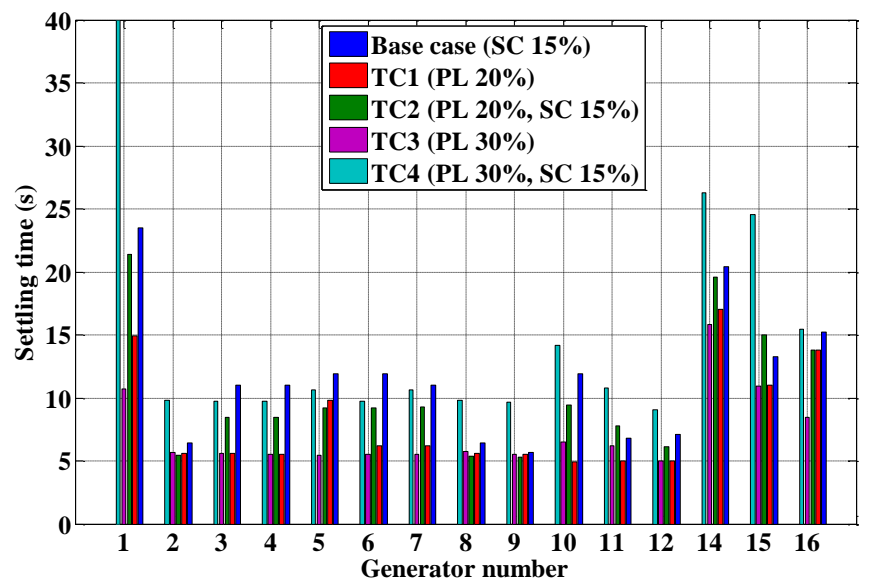

Figure 5. Generator settling time considering different DER penetration and decommissioning (TC1-TC4).

\section{B. Effect of DER location}

To study the effect of DER location four TCs based on previously described TC3 $\left(30 \% P L_{i}\right.$, no conventional unit decommissioning) are designed and the results for maximum rotor angle deviation are shown in Fig. 6. TC5 has 30\% $P L_{i}$ only in NETS area ( $0 \%$ in the rest of the system), while TC6 has $30 \% P L_{i}$ in the whole system except NETS area $\left(0 \% P L_{i}\right.$ for NETS). In a similar manner TC7, TC8 and TC9 consider also conventional generation decommissioning along with $30 \%$ DER penetration in the whole system, in NETS area and everywhere except NETS, respectively. It should be noted that the applied three phase fault still remains within NETS area (G1 terminals) for all TCs.

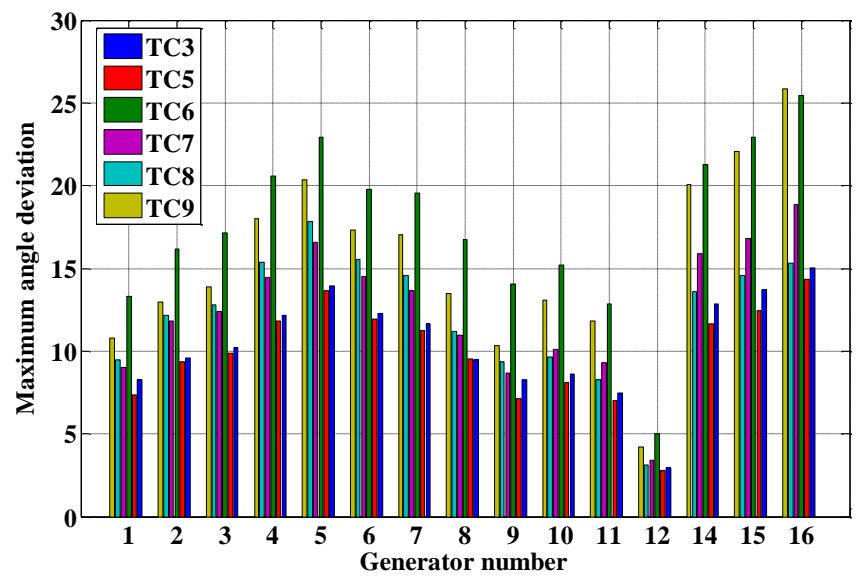

Figure 6. Maximum rotor angle deviation for different DER locations.

When DERs are connected only in NETS area of the system where the fault is also applied (TC5), the impact on the entire system is positive due to the de-loading of most of the conventional generators. In the case where there are no DERs connected to the area where the fault is applied (TC6), larger deviations in rotor angles are noticed for the whole system in similar amplitudes as to when no DERs at all are connected. This indicates a local effect of DERs inside the area where the fault is applied, which can either alleviate or worsen the stress to the rest of the network.

Considering TC7-TC9, where decommissioning of conventional generators is also taken into account, the results are similar. When DERs are connected and conventional units decommissioned in the NETS area slightly larger deviations compared to TC7 are observed within the area and smaller outside it. On the other hand, in the case when DER units are connected to the rest of the network except NETS, larger deviations are noticed for the entire system as before. In general, the positive or negative effect of DER penetration and decommissioning of conventional generation is a local effect and it can lead to improvement of transient responses in nearby areas, especially in the case when there are DERs within the area where the fault happens.

It is noted that the penetration of DERs within certain areas (mainly NETS and NYPS) can also change the power flows, especially on tie lines, which in turn affects the dynamic behavior of the system. Moreover, reduction in inertia of some generators in the system might have a more prominent effect than the reduction of inertia of the others.

\section{Effect of DER type}

The effect of the type of DERs is studied by changing the mix between Type 3 DFIGs and Type 4 FCC units. TC1 and TC2 that use the standard DER mix $(66.67 \%$ DFIGs and $33.33 \%$ FCC units), as in all previous cases, are now compared with TC10-TC13. TC10 that uses only DFIGs and TC11 that uses only FCC units are based on TC1 while TC12 and TC13 that use only DFIGs and only FCC units, respectively, are based on TC2 (inertia reduction considered). 


\section{$>$ ACCEPTED VERSION OF THE PAPER <}

By observing the maximum angle deviation shown in Fig. 7, FCC units tend to have more positive impact on system stability due to the fact that they are fully controllable and do not generally contribute to slow oscillating behaviour. This positive effect is not affected whether conventional generation is decommissioned or not.

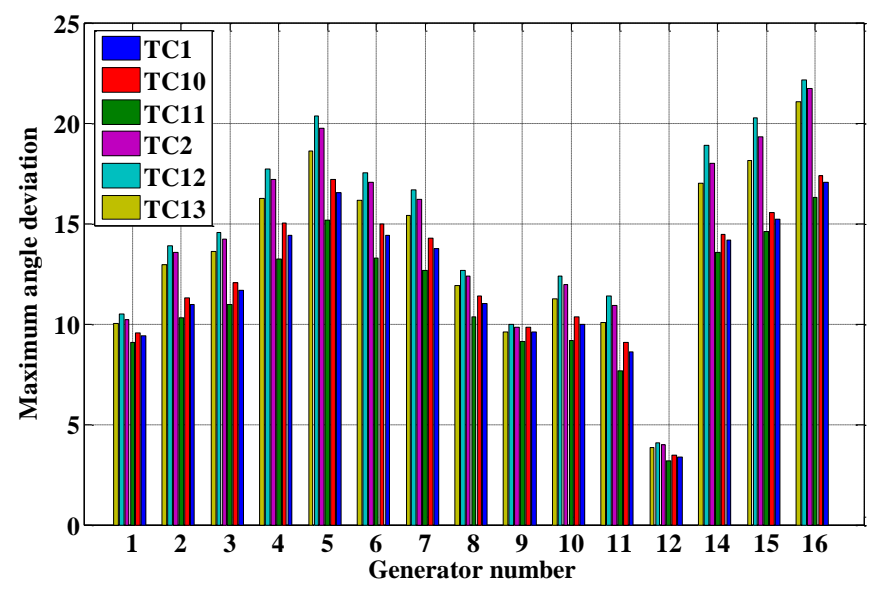

Figure 7. Maximum rotor angle deviation considering different DER mix.

\section{Overview of test cases}

As mentioned in Section II, the TSI is a measure of the system stability considering each specific contingency. The TSI values for the test cases studied are shown in Table I. While the maximum rotor angle deviation for each generator can provide an estimate of whether the specific generator is more stable or not, there are some cases where the maximum rotor angle deviation of most generators seems to drop and the TSI drops as well. This can happen because even if the maximum rotor angle deviation of the generators drops, the difference between two generator rotor angles at a certain point might still increase.

Examples that illustrate this are TC2 and TC4 where maximum angle deviation shown in Fig. 4 is smaller in TC4 indicating improvement in system stability, but the TSI drops slightly indicating the opposite effect. However, the settling time presented in Fig. 5 is consistent with the TSI. In general, the information provided by the three indices are complimentary, however, the TSI can provide a fast indication of whether the system becomes more or less stable when comparing different cases.

TABLE I. TSI FOR ALL EXAMINED CASES

\begin{tabular}{|c|c|c|c|}
\hline TC & TSI & TC & TSI \\
\hline Base Case & 62.51 & $\boldsymbol{T C 7}$ & 62.97 \\
\hline TC1 & 64.46 & $\boldsymbol{T C} 8$ & 64.9 \\
\hline $\boldsymbol{T C 2}$ & 61.74 & $\boldsymbol{T C 9}$ & 60.42 \\
\hline $\boldsymbol{T C 3}$ & 65.19 & $\boldsymbol{T C 1 0}$ & 64.13 \\
\hline $\boldsymbol{T C 4}$ & 61.38 & $\boldsymbol{T C 1 1}$ & 65.07 \\
\hline $\boldsymbol{T C 5}$ & 65.72 & $\boldsymbol{T C 1 2}$ & 61.37 \\
\hline $\boldsymbol{T C 6}$ & 57.85 & $\boldsymbol{T C 1 3}$ & 62.43 \\
\hline
\end{tabular}

\section{PROBABILISTIC APPROACH USING MONTE CARLO}

DERs interact with the system in many different ways, not only by altering the dynamic behavior of the system but also changing other parameters that can affect system stability, such as power flows and the inertia of synchronous generators when considering decommissioning of conventional units. Therefore, it is not straightforward, if possible at all, to define the impact of DERs by performing limited number of deterministic studies only.

\section{A. Effect of fault location and duration}

Monte Carlo simulations are carried out for TC1-TC3, TC5, TC6 for different fault locations and fault duration. Three phase faults are considered for all simulations as worst case scenarios. 1000 cases are simulated for each of the above mentioned TCs with the fault location varying randomly following a uniform distribution at all buses of the network and the fault duration following a normal distribution with a mean value of 14 cycles and standard deviation 0.33 cycles. The results are presented in Table II. The number of unstable cases (negative TSI) and the number of cases where at least one generator is still oscillating after $20 \mathrm{sec}$ are observed in order to ensure that the behavior analyzed above can be generalized.

\section{TABLE II. MONTE CARLO STUDIES}

\begin{tabular}{|c|c|c|}
\hline TC & $\begin{array}{c}\text { No of unstable } \\
\text { cases }\end{array}$ & $\begin{array}{c}\text { No of cases with } \\
\text { sustained oscillations }\end{array}$ \\
\hline Base Case & 612 & 7 \\
\hline TC1 & 62 & 29 \\
\hline TC2 & 234 & 365 \\
\hline TC3 & 11 & 11 \\
\hline TC5 & 88 & 912 \\
\hline TC6 & 422 & 226 \\
\hline
\end{tabular}

By comparing the base case, TC1 and TC3, it is seen that increasing penetration level of DER without decommissioning of conventional units decreases drastically the number of unstable cases but can cause a small increase in cases with sustained oscillations. When additionally decommissioning of conventional generators (TC2) is considered, the number of unstable cases increases drastically but is still smaller than the base case without DERs. However, under these circumstances a significant increase in cases with sustained oscillations is observed, which leads to the conclusion that reducing the system inertia can result in poor oscillation damping. In TC5 all examined cases lead to sustained oscillations for at least one of the generators and there is also a higher number of unstable cases noticed compared to TC3. This can be mainly attributed to the changes in power flows that resulted in lower damping of inter-area modes. By comparing the results of TC5 and TC6, it is evident that when DERs are added to the NETS area, the system tends to be more stable, especially considering non-oscillatory stability.

\section{B. Application of hierarchical clustering to determine generator grouping}

Monte Carlo simulations varying the fault location, fault duration and system loading are carried out to determine the patterns of generator groupings. Fault location is varying randomly considering all system buses, fault duration follows a normal distribution with mean value 13 cycles and standard deviation 0.67 cycles and system loading follows a normal distribution with a mean value of $1 \mathrm{pu}$ and standard deviation 


\section{$>$ ACCEPTED VERSION OF THE PAPER <}

$0.033 \mathrm{pu}$. The operating point of all conventional generators $\left(T C G_{i}\right)$ and DERs is scaled according to the system loading factor. 1000 cases are simulated and the generators are clustered into different groups based on the values of the rotor angles 100 cycles after the fault is cleared. The Euclidean distance is used to measure the similarity between clusters and the cutoff value to form the clusters is chosen as 360 degree, as in [4]. The base case and TC2 are used in the simulations and the most important groupings obtained are presented in Table III.

For the base case, 519 cases are unstable and 27 different groupings are identified within those cases, while for TC2, 176 cases are unstable and 9 groupings are obtained. Since the total number of unstable cases for the base case is larger, there are more generator groupings identified. However, apart from the 8 most important groupings shown in Table III, the rest occur only one or two times.

Considering only the important groupings, patterns 1-5 are identified in both cases, with 1, 3 and 5 being more frequent in TC2. More specifically, when DERs are added, G8, G9 and G11 tend to become more frequently unstable without interacting with other generators, compared to the base case. In pattern 2, all generators are grouped together, which means that at 100 cycles after the fault is cleared all generator rotor angles are still close and the unstable generators have not started to accelerate significantly yet. This is the most frequent pattern in the base case. Moreover, pattern 6 is only observable in TC2 and patterns 7 and 8 only in the base case. In general, the frequency as well as the number of the observed patterns is changing when DERs are connected. This means that some patterns might not be present when DERs are connected and at the same time, new patterns might appear after connecting DERs.

TABLE III. GENERATOR GROUPINGS

\begin{tabular}{|c|c|c|}
\hline Pattern & $\begin{array}{c}\text { No of contingencies } \\
\text { (Base case/TC2) }\end{array}$ & Grouping \\
\hline $\mathbf{1}$ & $8(4.5 \%) / 6(1 \%)$ & $(\mathrm{G} 1-\mathrm{G} 7, \mathrm{G} 9-\mathrm{G} 16) /(\mathrm{G} 8)$ \\
\hline $\mathbf{2}$ & $58(33 \%) / 324(62 \%)$ & $(\mathrm{G} 1-\mathrm{G} 16)$ \\
\hline $\mathbf{3}$ & $57(32 \%) / 61(12 \%)$ & $(\mathrm{G} 1-\mathrm{G} 8, \mathrm{G} 10-\mathrm{G} 16) /(\mathrm{G} 9)$ \\
\hline $\mathbf{4}$ & $4(2 \%) / 10(2 \%)$ & $(\mathrm{G} 1, \mathrm{G} 10-\mathrm{G} 16) /(\mathrm{G} 2-\mathrm{G} 9)$ \\
\hline $\mathbf{5}$ & $38(22 \%) / 42(7 \%)$ & $(\mathrm{G} 1-\mathrm{G} 10, \mathrm{G} 12-\mathrm{G} 16) /(\mathrm{G} 11)$ \\
\hline $\mathbf{6}$ & $6(3 \%) /-$ & $(\mathrm{G} 1-\mathrm{G} 3, \mathrm{G} 8-\mathrm{G} 16) /(\mathrm{G} 4-\mathrm{G} 7)$ \\
\hline $\mathbf{7}$ & $-/ 14(3 \%)$ & $(\mathrm{G} 1, \mathrm{G} 10-\mathrm{G} 16) /(\mathrm{G} 2, \mathrm{G} 3, \mathrm{G} 9) /(\mathrm{G} 4-\mathrm{G} 7)$ \\
\hline $\mathbf{8}$ & $-/ 20(4 \%)$ & $(\mathrm{G} 1-\mathrm{G} 9) /(\mathrm{G} 10-\mathrm{G} 16)$ \\
\hline
\end{tabular}

\section{CONCLUSIONS}

The impact of the connection of non-synchronous generation on the dynamic signature of power systems is investigated in this paper. Three indices (maximum angle deviation, TSI and settling time) are used to determine the effect of DERs, both DFIGs and FCC units, on non-oscillatory and oscillatory stability. A deterministic approach is initially followed by studying the effect of several factors, including the penetration level, the conventional generation decommissioning, the system loading conditions and the type and location of connected DERs. The three indices considered are complimentary with respect to the information they provide. While the maximum rotor angle deviation and the settling time can provide information on specific generators the TSI is a sufficient overall measure of system stability for a specific contingency.

Since there are a lot of uncertainties affecting system stability a probabilistic approach is followed to generalize the conclusions. Hierarchical clustering is also applied to investigate the changes in generator groupings after a fault, when DERs are connected to the network.. The identified groups are not significantly different, but the frequency of occurrence of certain groups is changing.

In general, connection of DERs has a positive effect on system stability when conventional units are not decommissioned. However, the amount and the location of decommissioned conventional generation has a significant adverse impact on system dynamic behaviour. Moreover, the connection of DERs, decommissioning and de-loading of conventional generators can change the power flows in the network, especially on tie lines, and therefore can affect system stability by causing prolonged oscillations in the system, though not necessarily system instability.

\section{REFERENCES}

[1] J. Quintero, V. Vittal, G. T. Heydt, Hui Zhang, "The Impact of Increased Penetration of Converter Control-Based Generators on Power System Modes of Oscillation," IEEE Trans. Power Syst., vol. 29, no. 5, pp: 2248-2256, Sept. 2014.

[2] S. Eftekharnejad, V. Vittal, G. T. Heydt, B. Keel, J. Loehr, "Impact of increased penetration of photovoltaic generation on power systems," IEEE Trans. Power Syst., vol. 28, no. 2, pp: 893-901, May 2013.

[3] T. Guo and J. V. Milanovic, "Probabilistic Framework for Assessing the Accuracy of Data Mining Tool for Online Prediction of Transient Stability," IEEE Trans. Power Syst., vol. 29, pp. 377-385, 2014.

[4] T. Guo and J. V. Milanović, "Identification of Power System Dynamic Signature Using Hierarchical Clustering", IEEE Power \& Energy Society General Meeting 2014, Washington, USA, 27-31 July 2014.

[5] WECC Wind Plant Dynamic Modeling Guidelines, WECC Renewable Energy Modeling Task Force, 2014.

[6] P. Sorensen, B. Andresen, J. Fortmann, P. Pourbeik, "Modular structure of wind turbine models in IEC 61400-27-1," IEEE Power and Energy Society General Meeting 2013, 21-25 July 2013.

[7] M. Ali, I. S. Ilie, J. V. Milanovic, G. Chicco, "Wind Farm Model Aggregation Using Probabilistic Clustering," IEEE Trans. on Power Syst., vol. 28, no. 1, pp: 309-316, Feb. 2013.

[8] WECC Solar Plant Dynamic Modeling Guidelines, WECC Renewable Energy Modeling Task Force, 2014.

[9] S. Eftekharnejad, V. Vittal, G. T. Heydt, B. Keel, and J. Loehr, "Small Signal Stability Assessment of Power Systems with Increased Penetration of Photovoltaic Generation: A Case Study," IEEE Trans. Sust. Energy, vol. 4, pp. 960-967, 2013.

[10] D. Gautam, V. Vittal, T. Harbour, "Impact of Increased Penetration of DFIG-Based Wind Turbine Generators on Transient and Small Signal Stability of Power Systems," IEEE Trans. Power Syst., vol. 24, no. 3, pp: 1426-1434, Aug. 2009.

[11] G. Rogers, Power System Oscillations. Norwell: Kluwer Academic Publishers, 2000.

[12] B. Pal and B. Chaudhuri, Robust Control in Power Systems. New York: Springer Inc., 2005.

[13] DIgSILENT-PowerFactory User Manual v15, DIgSILENT GmbH, 2014.

[14] P. Kundur, Power System Stability and Control. New York, NY, USA: McGraw-Hill, 1994.

[15] J. Fortmann, S. Engelhardt, J. Kretschmann, C. Feltes, I. Erlich, "New Generic Model of DFG-Based Wind Turbines for RMS-Type Simulation," IEEE Trans. On Energy Conversion, vol.29, no.1, pp.110118, 2014. 